EDUCATION

\title{
PSYCHOLOGY
}

I+D+i

Research, Innovation and Solutions on-line ${ }^{\circledR}$

\section{La reflexividad como competencia transversal en los estudios de psicología: límites y posibilidades en los entornos virtuales}

\section{Conchi San Martín Martínez ${ }^{1}$, Víctor Jorquera Fariñas ${ }^{2}$, Jordi Bonet i Martí}

\author{
${ }^{1}$ Departamento de Psicología Básica, Universidad de Barcelona, \\ Universitat Oberta de Catalunya \\ ${ }^{2}$ Departamento de Psicología Social, Universidad de Barcelona, Universitat Oberta \\ de Catalunya \\ ${ }^{3}$ Departamento de Métodos de investigación y diagnóstico en educación, \\ Universidad de Barcelona, Universitat Oberta de Catalunya
}

\section{España}

Dra.Conchi San Martín Martínez. Universidad de Barcelona, Dpto de Psicología Básica. Passeig de la Vall d'Hebron, 171. (Campus Mundet) Barcelona. C.P. 08035. Spain. E-mail: csan_martin@uoc.edu

(C) Education \& Psychology $\mathrm{I}+\mathrm{D}+\mathrm{i}$ and Editorial EOS (Spain) 


\section{Resumen}

En este artículo se pretende reflexionar sobre la necesidad y posibilidad de introducir una práctica docente facilitadora de la reflexividad del alumnado en el ámbito universitario, más concretamente en el ámbito de la disciplina psicológica. Esta reflexión toma en consideración los límites y posibilidades de implementar esta práctica en los incipientes entornos virtuales de enseñanza-aprendizaje y en el marco de los cambios propuestos en el nuevo espacio europeo de educación superior. De forma particular pretendemos poner en discusión: (a) cómo la reflexividad resulta una competencia fundamental a potenciar no sólo en el currículum del alumnado sino también en la práctica docente, cuestión que en parte se relaciona con las peculiaridades de la enseñanza de la disciplina psicológica y, (b) cómo ciertas características de los entornos virtuales de aprendizaje permiten constituir un espacio particularmente flexible para introducir e implementar las prácticas reflexivas.

Palabras Clave: Espacio Europeo Educación Superior, Competencias, Reflexividad, Entornos Virtuales de aprendizaje.

Recibido: 15/03/08 Aceptación inicial: 07/04/08 Aceptación definitiva: 05/05/08 


\begin{abstract}
Our aim in this paper is to consider the need and possibility to encourage university students' reflection through the teaching practice, particularly those studying the discipline of psychology. This study takes into consideration the limits and possibilities of implementing this practice in the incipient e-learning environments and also within the framework of the new European Space for Higher Education. We discuss two topics: (a) how reflective thought embodies a fundamental competency to be promoted both in the student's curriculum but also in teaching practice. This aspect relates partly to the peculiarities of the psychology discipline and, (b) how certain characteristics of e-learning environments constitute a particularly flexible space for introducing and implementing reflective teaching and learning practices.
\end{abstract}

Keywords: European Space for Higher Education, competencies, reflective teaching-learning, e-learning environments.

Received: 15/03/08 Initial Acceptance: 07/04/08 Final Acceptance: 05/05/08 


\section{Introducción}

Los cambios propuestos en el espacio europeo de educación superior (EEES) han supuesto un profundo replanteamiento de las metodologías de enseñanza y aprendizaje, muy especialmente al fomentarse un esfuerzo por delimitar las competencias y capacidades que debe adquirir y desarrollar el alumnado frente a estructuraciones más clásicas centradas en la adquisición de conocimientos específicos (MECD, 2003). La implementación de este viraje desde los contenidos hacia las competencias está suponiendo importantes cambios en el diseño y las prácticas docentes universitarias, rescatándose cuestiones como el fomento de la reflexividad como competencia a desarrollar (González, 2006). A su vez en este contexto de innovación en el espacio universitario emergen nuevas tecnologías, muy especialmente en lo referido al desarrollo de entornos virtuales de aprendizaje, que están tomando un lugar preeminente en la canalización de la transformación universitaria (Cabero, 2005). Sin embargo son también de notar ciertas dificultades relacionadas con la novedad, urgencia y, en ocasiones, fragmentariedad con que se han ido incorporando estas cuestiones.

Es por ello que, aunque conscientes de que la implantación de estos cambios no ha finalizado, creemos que existe suficiente trayectoria como para interrogarnos sobre dos cuestiones prácticas. En primer lugar la consideración de la reflexividad como una de las competencias fundamentales a potenciar en el crecimiento y formación del alumnado, de modo especial en el ámbito de la disciplina psicológica por sus particulares características curriculares y de proyección profesional, y también extensible a disciplinas afines. A nuestro entender, esta cuestión está necesariamente acompañada de un análisis sobre la necesidad de un cuestionamiento reflexivo sobre las propias prácticas docentes. En segundo lugar, proponemos analizar los límites y posibilidades de una práctica reflexiva en entornos virtuales de aprendizaje.

Con el ánimo de poner en práctica un primer ejercicio reflexivo tomamos como punto de partida nuestras propias prácticas profesionales como docentes en ámbitos presenciales, virtuales y semi-presenciales de diferentes universidades en el ámbito del estado Español para el análisis de dichas cuestiones. 


\section{Práctica docente reflexiva y reflexividad como competencia del alumnado: Dos caras de una misma moneda}

En este apartado analizamos en primer lugar la noción de reflexividad como competencia en el alumnado, considerando la notable y específica relevancia que toma esta cuestión en el ámbito concreto de la disciplina psicológica. En segundo lugar consideramos cómo la implementación de estrategias que fomenten un aprendizaje reflexivo no es posible sin el desarrollo de una práctica reflexiva por parte del docente. Esto, sin ser tarea fácil, nos resulta de especial interés y creemos que constituye la doble cara de una misma moneda.

Reflexividad, pensamiento reflexivo, indagación crítica, práctica reflexiva, pensamiento crítico, etc. son términos que, tal y como indica Phan (2006), se han empleado como sinónimos aún cuando éstos puedan suponer matices diferentes en la literatura especializada. En cualquier caso, la noción de reflexividad en las dinámicas de enseñanza y aprendizaje ha sido empleada mayormente para referir una amplia gama de procesos de pensamiento en estrecha relación al desarrollo de capacidades metacognitivas. Por ejemplo, para Badia y Monereo (2004) la capacidad reflexiva constituye una habilidad y competencia central en la promoción de una enseñanza estratégica, enseñanza basada en el principio de la construcción conjunta de significados y en la cesión progresiva del control en los procesos de enseñanza (Coll, 1985; Rogoff, 1993) que, en definitiva, apunta hacia un aumento de la conciencia del alumnado sobre su propio proceso de aprendizaje.

Excede los objetivos del presente trabajo ofrecer una revisión exhaustiva sobre la amplia literatura que ha tomado como eje el estudio de la reflexividad y su fomento, y donde cabe destacar trabajos clásicos como los de Mezirow (1981), Schön (1992) ó Zeichner y Liston (1991). Pese a ello sí es importante señalar cómo el fomento de la reflexividad puede entenderse ligado a diferentes niveles de impacto: desde una adquisición de aprendizajes significativos y habilidades específicas para adquirir una expertitud hasta la necesidad de preparar a estudiantes y profesores para que sean reflexivos con una prospectiva de cambio y transformación social.

La noción de reflexividad que aquí empleamos estaría en concordancia con la expuesta por Chacón (2006, p. 51), cuando señala: "Reflexionar implica un acto de pensamiento que nos 
permite abstraernos para observar, debatir consigo mismo y tratar de explicar nuestras acciones. Es mirar críticamente lo que se hace, justificar cada una de las decisiones tomadas y profundizar desde el cuestionamiento propio que nos permita rectificar o tomar decisiones que pretenden, en todo caso, mejorar nuestras prácticas". En este sentido existen diferentes herramientas útiles desde el punto de vista de su potencialidad en la generación de reflexividad. Por ejemplo, Medina (2007) nos ofrece una sistematización de procedimientos que pensamos pueden resultar de utilidad en el espacio universitario: el diálogo reflexivo, el modelaje metacognitivo, la traducción dialógica, el análisis de casos o el diario reflexivo. La idea básica de estos procedimientos es la de fomentar el "aprender a aprender" así como la implicación en un proceso de aprendizaje significativo. Sin embargo, no querríamos dejar de indicar, en acuerdo con los autores citados anteriormente, que si bien es necesaria una cierta planificación e implementación de estos procesos de toma de conciencia reflexiva ello no debe convertirse en un mero recetario de técnicas docentes o en una mera declaración de buenas intenciones.

Ahora bien, el fomento de la reflexividad como competencia creemos que tiene condicionantes específicos en función de la disciplina concreta que estemos abordando (Medina, 1999). Es por ello que, para los objetivos aquí planteados, nos interesa analizar cómo el planteamiento de la reflexividad como una meta a alcanzar en el desarrollo de las competencias básicas del alumnado de psicología debe tener presente una serie de particularidades, relativas a la propia estructura de la disciplina así como a su proyección profesional. Es este el aspecto que desarrollamos a continuación.

La importancia de la reflexividad como competencia transversal en el ámbito curricular de la psicología.

La complejidad que ha llegado a alcanzar la estructura curricular de la enseñanza de la psicología se debe en parte al hecho de que se trata de una disciplina multiparadigmática en el sentido que Kuhn (1971) otorgó al término paradigma. Esto implica la coexistencia de una diversidad de planteamientos teóricos, prácticos e ideológicos, de escuelas y de maneras de entender la tarea social de la psicología (Caparrós, 1984). Esta diversidad sólo parece asimilable a partir del desarrollo de una capacidad reflexiva por parte del alumnado, capacidad que posibilite 
adoptar diversos puntos de vista sobre una misma cuestión, conjugarlos, ponerlos en relación, considerar los límites y las potencialidades de cada uno de ellos, y finalmente tomar conciencia de que el compromiso con un punto de vista determinado implica la exigencia ética de defenderlo argumentalmente, evitando las tendencias a dar por cerrado un diálogo intradisciplinar, y por supuesto, interdisciplinar (Gordo y Linaza, 1996) que está siempre abierto. Esta capacidad reflexiva también debe permitir valorar cuál es el grado de conocimiento de que debe disponerse para emitir un juicio. Por tanto se trata de una capacidad abierta a un proceso de aprendizaje continuo (dado que no es un punto de llegada sino un punto de partida), respecto del cual el alumnado ha de desarrollar los criterios, habilidades e instrumentos para poder valorar y hacer balance sobre su progresión en él. Dicho en otros términos, la reflexividad debe fomentarse como competencia transversal y de manera progresiva en la formación académica.

La importancia de la reflexividad así entendida se multiplica en la medida en que la tarea profesional en el campo de la psicología estará marcada por la necesidad de una intervención conjunta con otros profesionales basada en este diálogo intra e interdisciplinar. De esta manera puede constituirse en una competencia que puede ayudar a establecer puentes entre la formación académica y la experiencia profesional (De la Fuente, Justicia, Casanova, y Trianes, 2005). En esta línea una de las cuestiones importantes a trabajar y desarrollar en el ámbito de la psicología y otras disciplinas relacionadas, es el proceso por el que se toma conciencia sobre la manera en que construimos nuestros conocimientos y/o actitudes sobre el "otro", es decir, ese otro diferente con el que más tarde el alumnado deberá relacionarse profesionalmente (Biglia y San Martín, 2005). Podemos decir que esta construcción del otro está atravesada por diversos factores. Por una parte, factores de aprendizaje formal, en función del contenido y orientación de las teorías $\mathrm{y}$ conceptualizaciones recibidas en el contexto académico, que como ya hemos indicado responde a una realidad multiparadigmática. Por otra parte también están los factores de experiencia personal, que las más de las veces no tienen cabida formalmente expresa en el proceso de formación. En este contexto la utilización de herramientas pedagógicas reflexivas como el denominado diario reflexivo (Medina, 2007) puede constituir una herramienta de gran potencial, que también resulta adaptable a diferentes objetivos de aprendizaje, en entornos presenciales pero también virtuales. Básicamente permite tomar la propia experiencia de aprendizaje como objeto de reflexión al posibilitar contrastar la evolución de los propios pensamientos y aprendizajes. En 
el fondo se trataría de una manera de potenciar una apropiación personal y dialogada de los conocimientos trabajados, más allá de un aprendizaje memorístico y acrítico, que también dé cabida a la expresión y toma en consideración de aspectos motivacionales y emocionales (Diotima, 1999), que van a tener un papel fundamental en la construcción de la relación profesional con el "otro".

¿Es posible promocionar una competencia reflexiva en el alumnado sin una práctica docente reflexiva?

Para poder llevar a cabo unas prácticas docentes que sean capaces de dar cuenta de la realidad multiparadigmática de la disciplina, de permitir "sacar a flote" los diversos factores implicados en la construcción del "otro" y, por tanto, capaces de animar al alumnado en el uso de las estrategias reflexivas como herramienta de aprendizaje, el profesorado debe poder también incorporar la reflexividad y transmitirla en nuestra docencia. Transmitirla con una actitud abierta que haga proliferar la diversidad de puntos de vista del alumnado mediante una escucha activa que posibilite llegar a una construcción conjunta de significados compartidos. Esto no resulta tarea fácil (Nunn, 1996) e implica evitar (o mejor, tomar conciencia) nuestras propias tendencias a cerrar diálogos, que en la disciplina han estado abiertos por años, con puntos de vista "absolutistas". De esta manera el diálogo argumentativo se convierte al tiempo en la condición y el efecto de la autorreflexividad (Mercer, 2001).

En este sentido, diversos autores y experiencias muestran la utilidad y necesidad de propiciar un enfoque constructivo en la formación de docentes (Badia y Monereo, 2004; Imbernón, 2000; Medina, 2007). Por ejemplo, Badia y Monereo (2004) aportan evidencias empíricas sobre cómo el aumento del conocimiento explícito del profesorado sobre la enseñanza estratégica antes reseñada tiene una relación directa con la calidad de la aplicación de la secuencia instruccional en la práctica docente. El enfoque constructivo establece un contexto de enseñanza-aprendizaje en la formación de docentes donde la tarea fundamental del formador es proporcionar guías y ayudas que faciliten al profesorado la reflexión y/o investigación sobre su propia práctica docente. De esta manera, los citados autores afirman que: "el profesorado más que introducir innovaciones diseñadas por expertos, necesita desarrollar sus conocimientos previos apropiándose de contenidos relevantes, en paralelo a la transformación de sus prácticas 
educativas, a través de la reflexión sobre su práctica docente cotidiana, y la adquisición de un lenguaje que le permita interpretar esta práctica”(Badia y Monereo, 2004, p. 68).

Por su parte Medina (2007) muestra con claridad, a partir de la implementación de talleres para la práctica docente reflexiva, que la propia experiencia docente (los límites, posibilidades, contradicciones, etc.) debe ser el punto de partida ineludible para generar la reflexividad. Por ejemplo el recurso del diario reflexivo, antes señalado en el ámbito del alumnado, también resulta de utilidad para una formación docente reflexiva. Este procedimiento permite hacer visibles (para quien lee o escucha) las razones o justificaciones que sustentan el trabajo propuesto por el equipo docente. Es decir, se trata de razones que suelen formar parte de la reflexión interna docente y que lo llevan a unas elecciones, y no a otras, sobre contenidos, enfoques, herramientas y /o objetivos. De fondo está la consideración de que no sólo se están traspasando unos contenidos sino también unas maneras de aproximarse e interpretar la historia de la disciplina, así como unas actitudes. Tal y como ya se ha indicado, estos aspectos, en disciplinas como la psicología, pueden introducir un cierto conflicto, pero también riqueza, dado que son posibles aproximaciones muy diversas -ideológicas, teóricas, etc.- que si permanecen implícitas, y sin poder ser discutidas, pueden acabar configurando un currículum oculto, que creemos, vale la pena desvelar.

\section{Posibilidades y límites del espacio virtual para la práctica reflexiva}

Venimos, pues, definiendo la reflexividad como una competencia que surge de las prácticas dialógicas, como una habilidad que primero se elabora colectivamente para posteriormente poder ser desarrollada activamente en la subjetividad del alumnado y del profesorado en forma de diálogo interno. A continuación queremos reflexionar sobre los límites y posibilidades asociadas al uso de entornos virtuales de aprendizaje (EVA) para el desarrollo de las prácticas reflexivas en el ámbito de la docencia universitaria (Cabero, 2002). Si bien consideramos que los EVA expanden nuestras posibilidades de organización de la práctica docente y permiten una mayor agencialidad del estudiante, también queremos subrayar que estas posibilidades en ocasiones no son suficientemente aprovechadas convirtiéndose en límites que pueden llegar a condicionar el conjunto del proceso. Iniciamos este apartado evidenciando qué límites hemos detectado a partir de nuestra propia práctica docente en estos entornos, para posteriormente pasar a plantear de forma propositiva las posibilidades que nos abren como 
espacios para implementar procesos de reflexividad, y acabar identificando aquellos recursos que puedan facilitar esta reflexividad.

\section{Límites}

\section{Confusión entre información y conocimiento}

La infoesfera se ha convertido en uno de los depósitos de información más grandes creado por la sociedad humana. En este sentido, el aprendizaje virtual puede beneficiarse de este hecho en tanto que se multiplica exponencialmente la accesibilidad a las fuentes, manteniendo como único requisito técnico el contar con un punto de acceso (Wolton, 2003). Sin embargo, debe considerarse que una parte importante de la información disponible en las fuentes virtuales es imprecisa cuando no errónea, convirtiéndose así en una posible fuente de confusión (la denominada intoxicación).

A partir de nuestra experiencia docente consideramos que esta cuestión, a pesar de tratarse de un recurso externo a la formación, afecta enormemente a las posibilidades de desarrollar una práctica reflexiva en el aula. Frecuentemente el alumnado emplea acríticamente esta información para realizar las actividades de evaluación sin realizar un esfuerzo analítico para evaluar la fiabilidad (Comas y Sureda, 2007). No nos referimos únicamente a la práctica fraudulenta de copiar literalmente trabajos o fragmentos de textos encontrados en internet, sino al hecho de considerar como válidas fuentes de dudosa procedencia que, como se ha indicado, en ocasiones contienen imprecisiones y /o errores importantes. Parece que pervive la creencia de que por el hecho de estar accesibles en internet estas informaciones ya pueden ser consideradas como válidas. Tal vez, una de las causas que facilita esta situación es la confusión entre información y conocimiento, derivada de la herencia de un modelo de aprendizaje clásico centrado en la memorización de contenidos. El desarrollo del conocimiento, a diferencia de la adquisición de información, implica movilizar capacidades vinculadas al razonamiento, la argumentación, la contrastabilidad y el pensamiento crítico, todas ellas asociadas al desarrollo de prácticas reflexivas que permitan extraer conocimiento a partir del contraste y valoración de fuentes de información diversas. Esta cuestión resulta de especial relevancia en la propia actualidad de la disciplina psicológica, donde la diversidad de criterios epistemológicos habidos para el 
establecimiento del conocimiento legítimo está adquiriendo una complejidad nunca vista hasta la fecha. En este contexto el alumnado debe poder llegar a asumir la tarea de discernir e interrogar críticamente los criterios de legitimidad, de forma primero guiada y posteriormente autónoma, dejando a un lado el modelo de adquisición pasiva de información, donde la autoridad del texto escrito era incuestionada.

\section{Individualización del proceso de aprendizaje}

Abordamos a continuación no un condicionante externo, como en el punto anterior, sino un elemento referido a la propia dinámica de las relaciones que se establecen en un EVA. Un EVA es en sí mismo un sistema organizado que se integra en un sistema más amplio, y las sinergias que se producen entre los diferentes componentes constituyen una de las principales fuentes de producción de conocimiento. El aprendizaje en este entorno tiene la potencialidad de ayudar al alumnado a superar la relación biyectiva con el docente, y articularse con todos los componentes que forman parte del EVA. Sin embargo, en ocasiones, la falta de copresencialidad tiende a difuminar esta visión de colectividad y, de nuevo, a circunscribir e individualizar la relación comunicativa en el par docente-alumno. Esta relación dificulta el proceso de reflexividad en tanto que puede no sentirse partícipe del entorno organizativo donde se desarrolla su aprendizaje y, tal como hemos comentado en la primera parte, la reflexividad es promovida precisamente por la multiplicación de puntos de vista sobre un mismo asunto. Además, en el futuro profesional del alumnado de psicología se encuentran grandes desafíos en la constitución de equipos de trabajo diversos y multidisciplinares, cuyo funcionamiento se ve precisamente impedido por dinámicas de individualización.

\section{Dificultades de establecer una escritura y comunicación académica de calidad en un EVA}

Dado que la comunicación en espacios virtuales se realiza mayoritariamente de manera escrita, excepto en los casos en que se habilita una videoconferencia, la habilidad narrativa adquiere en estos espacios un rol fundamental para el desarrollo de los objetivos docentes, y de manera más concreta, para la adquisición de la reflexividad (Gee, 1996). Sin embargo, percibimos en ocasiones un mal uso de la escritura en los entornos virtuales académicos que empobrece la interacción comunicativa y afecta a la consecución de los objetivos de aprendizaje. A partir de 
nuestro trabajo docente en los EVA hemos evidenciado una tendencia bascular entre el empleo de la informalidad propia de otras plataformas de comunicación virtual (chats, mensajería de correo electrónico) y la excesiva formalidad asociada al correo epistolar. En este sentido, se evidencia que aún no se han desarrollado las competencias para promover un uso adecuado del discurso escrito así como un estilo argumentativo parsimonioso, razonado, reflexivo y crítico. Cabe señalar también cómo un empleo acrítico de la escritura afecta a las propias dinámicas de interacción: la organización de un debate en EVA donde aparece una acumulación de mensajes redundantes dificulta que pueda extraerse el sentido de la comunicación y desmotivan su lectura íntegra. La falta de competencias en el desarrollo de una escritura académica, así como en el buen uso de las herramientas de comunicación, puede convertirse en otro límite para la potenciación de la reflexividad (Medina, 2007).

\section{Oportunidades}

Tras explicitar las que consideramos principales limitaciones en los EVA a partir de nuestra propia práctica docente, pasamos a destacar qué oportunidades nos abren estos entornos en relación al fomento de la reflexividad.

1. Centrar el aprendizaje en el conocimiento y capacidades a partir de una organización crítica y reflexiva de la práctica docente

Como docentes que hemos desarrollado nuestro aprendizaje mayoritariamente en universidades presenciales, con un modelo enfocado a la adquisición de contenidos, tendemos aún a plantear nuestra práctica (docencia y evaluación) centrándonos en los contenidos y no en el desarrollo de capacidades (Sánchez, 2001). Como hemos venido señalando necesitamos replantear las actividades docentes, a partir de un nuevo paradigma de aprendizaje donde se facilite al alumno las herramientas necesarias para el desarrollo de sus capacidades, en especial las referidas a la práctica reflexiva. Si bien estas cuestiones también se han reactivado como reto en los entornos de enseñanza presencial, los EVA suponen la posibilidad de disponer de nuevas herramientas. Por ejemplo, podemos ayudar a diferenciar la información del conocimiento a partir del diseño de actividades críticas y reflexivas, o de la puesta en práctica de procesos participativos y dinámicos de revisión y debate sobre los ejercicios realizados. En este sentido, 
estimular debates sobre las dificultades que el alumnado tiene o ha tenido para la realización de una actividad, o para el estudio de un material, puede resultar de especial interés.

\section{Establecer sinergias entre los diferentes componentes del EVA para romper con las dinámicas} de individualización

Como docentes no podemos olvidar que el aula no es la suma agregada de estudiantes sino que constituye un sistema organizado, un espacio de interacción. En este sentido debemos favorecer y facilitar dinámicas de interacción en el aula, gestionando los posibles conflictos que puedan aparecer, favoreciendo un uso parsimonioso de la interacción, facilitando que se entienda que el aula es un espacio integrado donde se pueden plantear dudas y compartir procesos de aprendizaje participando en las actividades propuestas. Los entornos EVA introducen una importante flexibilidad al respecto de estas cuestiones (Duart y Lupiáñez, 2005). Sin embargo, no debe olvidarse que es importante estar respaldados en nuestras decisiones docentes por la institución en la que trabajamos, con lo que creemos necesario que se desarrollen protocolos de dinámicas a seguir y que se dé una total transparencia no sólo en las relaciones entre docentes y estudiantes, sino también entre docentes e institución.

3. El fomento de la escritura como práctica reflexiva y como herramienta de comunicación virtual.

Tal y como se ha señalado anteriormente el hecho de tener que escribir y leer las contribuciones que se realizan en el aula de los EVA, permite al alumnado desarrollar su capacidad crítica y reflexiva. A diferencia de la comunicación oral-presencial que puede resultar efímera, la comunicación por escrito nos da la posibilidad de repasar las intervenciones propias y ajenas en los mensajes que quedan registrados en el EVA. Esto permite al alumno reinterpretar los contextos comunicativos en función de lo que ha ido aprendiendo y puede convertirse en un elemento de captación del propio proceso de aprendizaje. Respecto al profesorado, este registro de la información puede constituirse a su vez en una herramienta útil, no sólo para realizar un seguimiento del progreso del alumnado, sino también para auto-evaluar la práctica docente.

\section{Recursos}


Finalmente a partir de los límites y oportunidades señalados hemos considerado conveniente apuntar aquellos recursos que a nuestro entender podrían servir para mejorar una práctica docente reflexiva, así como facilitar el proceso de aprendizaje.

\section{La adecuación de los materiales didácticos para el desarrollo de las capacidades reflexivas}

Hemos constatado que con cierta frecuencia los materiales didácticos empleados en un EVA tienden a seguir un modelo bastante parecido al empleado en los procesos de aprendizaje presencial. En este sentido consideramos que la selección, elaboración y organización de los materiales didácticos de las diferentes asignaturas ha de estar orientada hacia la promoción de la capacidad reflexiva, incidiendo de manera clara en la necesidad de contrastar diferentes fuentes, de adquirir el hábito de la lectura crítica, de favorecer la conexión con situaciones que formen parte del mundo que rodea al estudiante, así como de su futura carrera profesional. Esta problemática puede ser solucionada de manera autónoma por diferentes instituciones que utilizan los EVA. Ahora bien, otro gran límite, que necesitaría una solución más global, está relacionado con las licencias de propiedad intelectual de los materiales empleados que impiden, frecuentemente, la utilización de artículos académicos para la práctica docente. En este sentido consideramos necesaria la expansión de licencias que garanticen un libre acceso, como la Creative Commons, en la producción académica.

\section{La adecuación de las pruebas de evaluación a las competencias y no a los contenidos}

En este contexto las pruebas de evaluación deben estar dirigidas a valorar la adquisición de competencias por parte del alumnado En este sentido creemos que es necesario unificar criterios en vistas al diseño de pruebas de evaluación que pongan énfasis en el desarrollo de estas capacidades, y ofrecer recursos al profesorado para la elaboración de estas pruebas. Por otra parte, es necesario un replanteamiento del mismo concepto de pruebas de evaluación que pueda ir más allá del clásico formato textual, más en un contexto universitario donde se promueve la evaluación continuada. Sin embargo, para poder implementar este cambio es necesario que se reconozca la gran cantidad de trabajo docente que esto requeriría. Sólo así será posible poner las bases para acciones de evaluación más globales respecto de las capacidades demostradas por el

alumnado (por ejemplo, participación en debates, ejercicios que evalúen la evolución del 
aprendizaje en lugar de sus productos finales, etc.).

3. La habilitación de espacios virtuales y presenciales de debate transversal entre docentes para fomentar la reflexividad

Creemos que para promover la transversalidad en el fomento de la reflexividad a lo largo de los estudios de psicología sería positivo habilitar espacios de debate transversales donde docentes de diferentes asignaturas pudieran compartir sus experiencias, unificar criterios y proponer elementos educativos para el trabajo de la reflexividad; por ejemplo, a través del uso de plataformas de e-learning como Moodle o Claroline basadas en software libre y que integran utilidades como foros de debate, espacios de compartición de archivos y páginas editables.

\section{La capacitación y formación de los docentes en la didáctica de los entornos virtuales}

Como docentes en una campo relativamente nuevo como es el e-learning, abierto a constante evolución, resulta necesario adquirir recursos formativos y didácticos adecuados a las nuevas necesidades y retos (Duart y Lupiáñez, 2005). En este sentido consideramos que sería necesario diseñar y promover un itinerario formativo integral dirigido a las personas encargadas de la docencia en EVA para mejorar la capacitación en estrategias didácticas. Las instituciones académicas, y específicamente las que se dedican a una enseñanza virtual, han de tomar la encomiable decisión de poner en práctica estas cuestiones a riesgo de quedar obsoletas si no lo hacen.

\section{La elaboración de herramientas docentes dirigidas a un buen uso de la red de internet}

La red de internet debiera servir tanto a estudiantes como a docentes como fuente de recogida de información, estructurándose los recursos y determinando su fiabilidad. No nos referimos únicamente a los recursos disponibles en las colecciones digitales de las bibliotecas sino también a la elaboración de guías escritas que faciliten el uso crítico y reflexivo de la red.

\section{Establecer procesos específicos que fomenten la escritura académica crítica y reflexiva}


Consideramos que a pesar de tratarse de una competencia transversal en el itinerario de aprendizaje, dada la importancia de la escritura académica crítica y reflexiva sería necesario introducir núcleos de estudio donde se trabaje sustantiva y centralmente esta competencia

\section{Conclusiones}

El Espacio Europeo de Educación Superior implica nuevos desafíos para la práctica docente universitaria, entre los cuales está su nuevo énfasis en el aprendizaje de competencias frente al clásico aprendizaje de contenidos. A partir de la experiencia de nuestra práctica docente en el ámbito disciplinar de la psicología, tanto en universidades presenciales como virtuales, hemos valorado que una de las competencias que requiere el desarrollo integral del alumnado de psicología es la reflexividad.

La reflexividad, como proceso por el que el alumnado toma conciencia creciente de su propio proceso de aprendizaje y en el que el objetivo fundamental es la adquisición de herramientas de aprendizaje autónomo y crítico, es de vital importancia en las prácticas de enseñanza-aprendizaje de la disciplina psicológica. Esto es así debido al carácter multiparadigmático de la disciplina, pero también por la relevancia que adquieren los aspectos motivacionales y afectivos en el aprendizaje y desarrollo de lo que hemos llamado la relación con el "otro", ese otro diferente con el que el alumado de psicología mantendrá en el futuro una relación profesional. El desarrollo de la reflexividad permite al alumnado tomar conciencia crítica de cómo los propios afectos y actitudes personales acaban jugando un papel en la construcción de la relación con el otro, dado que éste no puede resultar ser tan sólo el objeto de la intervención psicológica en sentido estrictamente técnico, sino que el desarrollo de una relación profesional con el otro moviliza inevitablemente aspectos afectivos propios que no suelen trabajarse en el clásico sistema de aprendizaje centrado en contenidos.

Sin embargo, creemos que no es posible promover la reflexividad en el alumnado sin que seamos capaces a la vez de desarrollar un cuestionamiento reflexivo con respecto a nuestra práctica docente. Esta mirada reflexiva sobre nuestras prácticas debe llevarnos a una explicitación, a través de un diálogo argumentativo en el aula, de los criterios que fundamentan nuestras decisiones a la hora de confeccionar un determinado recorrido curricular que, en el caso 
de la psicología, se trata siempre de una elección entre otras posibles, y someter así a discusión pública dichos criterios para evitar de este modo que configuren un currículo oculto.

Finalmente, hemos tratado de explorar, desde nuestra propia experiencia docente, cuáles son los límites y posibilidades que nos ofrecen los entornos virtuales de aprendizaje con relación al desarrollo de la reflexividad. Hemos propuesto que, a partir de la aplicación de la reflexividad a nuestra forma de organizar la docencia en un EVA, podemos tomar conciencia de los elementos que en éste obstaculizan la reflexividad, como la confusión entre información y conocimiento, la individualización del proceso de aprendizaje y las dificultades que a veces se dan para una escritura académica y rigurosa. Los EVA contienen también la potencialidad para contrarrestar los efectos de estas dinámicas, ofreciéndonos por ejemplo la posibilidad de centrar la evaluación en competencias y no contenidos, herramientas para trabajar el diálogo argumentativo, la contrastabilidad y el pensamiento crítico, oportunidades para establecer sinergias entre el alumnado de forma que usen el EVA como espacio para compartir el propio proceso de aprendizaje y construir colectivamente el conocimiento compartido, y finalmente su énfasis en la escritura como promotora de reflexividad. También nos ofrece la posibilidad de constituirse en plataforma de intercambio de experiencias para el profesorado.

En la línea de las reflexiones planteadas por Sánchez (2001) creemos que los cambios que se han de realizar en el marco del EEES pueden llegar a buen término sólo si las instituciones académicas y las otras instituciones implicadas en los procesos formativos son capaces de entender que estas transformaciones no pueden depender del voluntarismo del profesorado sino que se han de enmarcar en un proceso de trabajo reconocido.

No querríamos finalizar sin expresar nuestro deseo de que los elementos aquí esbozados puedan ser objeto de reflexiones colectivas más generalizadas. 


\section{Referencias}

Badía, A. y Monereo, C. (2004). La construcción de conocimiento profesional docente. Análisis de un curso de formación sobre la enseñanza estratégica. Anuario de Psicología, 1, 47-70.

Biglia, B. y San Martín, C. (2005). La creació de l'altra a la investigació psicosocial. En AA.VV. Col.lectiu Investigacció (Eds.). Recerca activista $i$ moviments socials. Barcelona: Fundación Jaime Bofill (pp. 35-46).

Cabero, J. (Dir.) (2002). Las TIC y la Universidad. Sevilla: MAD.

Cabero, J. (2005). Las TICs y las universidades: retos, posibilidades y preocupaciones. Revista de Educación Superior, 34(3), 77-100.

Caparrós, A. (1984). La psicología y sus perfiles. Barcelona: Barcanova.

Chacón, M (2006). La enseñanza reflexiva en la formación de los estudiantes de pasantías de la carrera de Educación Básica Integral. Tesis doctoral no publicada. Universitat Rovira i Virgili, Tarragona. Recuperado 23 de abril de 2008 desde http://www.tesisenxarxa.net/

Coll, C. (1985). Acción, interacción construcción del conocimiento en situaciones educativas. Anuario de Psicología, 33, 59-70.

Comas, R. y Sureda, J. (2007). Ciber-Plagio académico. Una aproxiamación al estado de los conocimientos. Revista TEXTOS de la Cibersociedad, 10. Consultado en: http://www.cibersociedad.net, 26 de marzo de 2007.

De la Fuente, J., Justicia, F., Casanova, P. F., y Trianes, M. V. (2005). Perceptions about the construction of academic and professional competencies in psychologists. Electronic Journal of Research in Educational Psychology, 3(1), 3-34.

Diotima (1999). El perfume de la maestra. En los laboratorios de la vida cotidiana. Barcelona: Icaria.

Duart, J.M., y Lupiáñez, F. (2005) Estrategias en la introducción y uso de las TIC en la universidad. Revista de la Universidad y Sociedad del Conocimiento, 1, 5-32.

Gee, J. P. (1996). Social Linguistics and literacies. Ideology in discourses. Londres: Taylor y Francis.

González, I. (2006). Dimensiones de evaluación de la calidad universitaria en el espacio europeo de educación superior. Electronic Journal of Research in Educational Psychology, 10(4), 445-468.

Gordo, A. J. y Linaza, J. L. (eds.) (1996). Psicologías, discursos y poder (PDP). Madrid: Visor. 
Imbernón, F. (2000). La formación docente del profesorado universitario entre la realidad, el deseo y la utopía. I Congreso Internacional: Docencia universitaria e Innovación. Barcelona, Junio.

Kuhn, T. (1971). La estructura de las revoluciones científicas. México: FCE.

MECD (2003). La integración del sistema universitario español en el Espacio Europeo de Ensañanza Superior. Madrid: MECD.

Medina, J. L. (1999) La pedagogía del cuidado: saberes y prácticas en la formación universitaria en enfermería. Barcelona: Laertes.

Medina, J. L. (2007). Aprendizaje reflexivo: la enseñanza reflexiva en el seno del EEES (documento de trabajo).

Mercer, N. (2001). Palabras y mentes: cómo usamos el lenguaje para pensar juntos. Barcelona: Paidós

Mezirow, J. (1981). A critical theory of adult learning and education. Adult Education Quaterly, 32(1), 3-24

Nunn, C. (1996). Discussion in the college classroom. Journal of Higher Education, 67(3), 243265.

Phan, H. P. (2006). Examination of student learning approaches, reflective thinking, and epistemological beliefs: a latent variables approach. Electronic Journal of research in Educational Psychology, 10, 4(3), 577-610.

Rogoff, B. (1993) .Aprendices del pensamiento: el desarrollo cognitivo en el contexto social. Barcelona: Paidós.

Sánchez, J.A. (2001). El desarrollo profesional del docente universitario. Revista Universidades, 22. Consultado en: http://www.udual.org/CIDU/Revista/22/contenido.htm, 5 de enero de 2008.

Schön, D. (1992). La formación de profesionales reflexivos: hacia un nuevo diseño de la enseñanza y el aprendizaje en las profesiones. Barcelona: Paidós-Centro de publicaciones del Ministerio de Educación y Ciencia.

Zeichner, K. y Liston, D. (1991). Teacher education and the social conditions of schooling. New York: Routledge

Wolton, D. (2003). La otra mundialización. Los desafios de la cohabitación cultural global. Barcelona: Gedisa 
Conchi San Martín Martínez et al.

[Página en blanco por razones de paginación] 